\title{
Triptolide inhibits neutrophil extracellular trap formation
}

\author{
Haiyu Guan $^{1 \#}$, Lifen Xie ${ }^{1 \#}$, Zhenzhen $\mathrm{Ji}^{1}$, Rui Song ${ }^{2}$, Jieying $\mathbf{Q i}^{1}$, Xiaoli Nie ${ }^{1}$ \\ ${ }^{1}$ Department of Nephrology, Integrated Hospital of Traditional Chinese Medicine and Western Medicine, Southern Medical University, Guangzhou, \\ China; ${ }^{2}$ Department of Rheumatology, The Third Affiliated Hospital of Southern Medical University, Guangzhou, China \\ Contributions: (I) Conception and design: H Guan, L Xie; (II) Administrative support: Z Ji; (III) Provision of study materials or patients: R Song; (IV) \\ Collection and assembly of data: J Qi; (V) Data analysis and interpretation: H Guan, L Xie; (VI) Manuscript writing: All authors; (VII) Final approval \\ of manuscript: All authors. \\ \#These authors contributed equally to this work. \\ Correspondence to: Xiaoli Nie. Integrated Hospital of Traditional Chinese Medicine and Western Medicine, Southern Medical University, 13 \\ Shiliugang Road, Guangzhou 510315, China. Email: nx1117@163.com.
}

Background: Triptolide (PG490), as a triterpene dicyclic oxide has been reported to increase the generation of reactive oxygen species (ROS) and nitric oxide (NO) and induce apoptosis of RAW 264.7 cells in a dose-dependent manner. The activity of death NETs plays an important role in anti-bacterial processes in the human body. This study aimed to investigate the effect of triptolide (PG490) on neutrophil extracellular traps (NETs) formation.

Methods: After isolating peripheral blood neutrophils from healthy volunteers, cells were incubated with PG490 to observe and detect the level of NETs and detect the level of reactive oxygen species (ROS). The cells were cultured, stained and analyzed by fluorescence microscopy.

Results: Compared with the 12-myristate-13-acetate (PMA) group, the average fluorescence intensity of SYTOX Green in the PG490 + PMA group, as detected by a multifunctional microplate reader, was significantly decreased. Intracellular ROS were labeled by fluorescence, with fluorescence intensity then measured by multifunctional microplate reader and flow cytometry. The results showed that compared with the control group, the fluorescence intensity of the PMA group was significantly increased, while there was no significant difference between PMA group and PG490 + PMA group.

Conclusions: The production of NETs is inhibited by PG490 in vitro, which is not associated with the level of cellular ROS. This suggests that PG490in Tripterygium wilfordii Hook F can suppress related diseases.

Keywords: Triptolide; neutrophils; neutrophil extracellular traps (NETs); reactive oxygen species (ROS)

Submitted Jun 24, 2021. Accepted for publication Aug 06, 2021.

doi: 10.21037/atm-21-3522

View this article at: https://dx.doi.org/10.21037/atm-21-3522

\section{Introduction}

Tripterygium wilfordii Hook F, as a traditional Chinese medicinal herb, has been considered to have the functions of clearing away heat and detoxification, dispelling wind and clearing the collaterals, relaxing muscles, and promoting blood circulation. Recently, increasing studies have found that Tripterygium wilfordii Hook F has multiple functions including immunosuppressive (1), anti-inflammatory, antitumor activities (2), and regulation of autophagy (3). Triptolide (PG490), as a triterpene dicyclic oxide isolated from Tripterygium wilfordii Hook F, has been reported to increase the generation of reactive oxygen species (ROS) and nitric oxide (NO) and induce apoptosis of RAW 264.7 cells in a dose-dependent manner $(5-25 \mathrm{ng} / \mathrm{mL})(4)$.

A study on PG490-associated hepatotoxicity showed that pre-treatment with triptolide sensitized the liver to the 
stimulation of lipopolysaccharide (LPS), which can promote liver cell death by influencing programmed necroptosis (5). Generally, triptolide plays a vital role in cell death.

Neutrophils are the most abundant white blood cells in the blood, and when foreign pathogens and tissue damage occur, they are the first responders of the innate immune system (6). Neutrophils play a key role in the first line of immune defense against invading pathogens, they can migrate to the site of inflammation quickly to prevent the spread of pathogens, and their mechanisms of defense include phagocytosis of pathogens and lysozyme degranulation $(7,8)$. Studies have shown that (9), contrary to most phagocytes, neutrophils can also "depolymerize" their DNA and release it outside the cell to form a network structure called neutrophil extracellular traps (NETs). These reticular chromatin structures are based on DNA, inlaid with histone $(\mathrm{H})$, myeloperoxidase (MPO), neutrophil elastase (NE), and cathepsin G (CG), protease 3 (PR3), and other proteins that can kill bacteria, increase cell permeability, and isolate and kill microorganisms (10). The activity of death NETs plays an important role in anti-bacterial processes in the human body. However, studies have shown that NETs are found in a variety of autoimmune diseases such as systemic lupus erythematosus (SLE) (11), rheumatoid arthritis (RA) (12), and antineutrophil cytoplasmic antibody (ANCA)-associated vasculitis (AAV) (13), and the high production of NETs leads to excessive inflammatory cell death, mass release of inflammatory factors, and then promotes the production of inflammatory response (14). Elevation of the levels of ROS is considered to play a key role in NETs formation (15).

Low levels of oxidative stress can help cells kill invading pathogens, but excessive levels of oxidative stress can also cause cell death. Studies have shown that triptolide inhibited the Nrf2-driven glutathione synthesis pathway and led to apoptotic changes in IDH1-mutated cancer cells via redox catastrophe (16). However, limitations such as the levels of triptolide-induced NETs and ROS in human neutrophils, mean that further investigation is required. Therefore, different from an animal model study by Huang et al. (17), our study aimed to observe the effect of triptolide on the NETs and ROS levels of human neutrophils, and explore the anti-inflammatory and antirheumatic mechanism of triptolide-induced neutrophils. We present the following article in accordance with the MDAR reporting checklist (available at https://dx.doi. org/10.21037/atm-21-3522).

\section{Methods}

\section{Samples}

Healthy adults were recruited as volunteers, including those who had no history of smoking, no recent trauma, no surgery, no skin ulcers, no diabetes, no cardiovascular disease, no blood system diseases, and other factors that affect NETs. After informed consent had been provided by the participants, their peripheral blood samples were collected $(10 \mathrm{~mL} / \mathrm{sample})$. All procedures performed in this study involving human participants were in accordance with the Declaration of Helsinki (as revised in 2013). The study was approved by ethics committee of Integrated Hospital of Traditional Chinese Medicine and Western Medicine (No.: NFZXYEC-201906-Y1) and informed consent was taken from all the patients.

\section{Drugs and reagents}

The following drugs and reagents were procured for use in this study: triptolide (Dalian Meilun Biological Technology Co., Ltd., Dailan, Liaoning, China); human neutrophils separation solution (Tianjin Haoyang Biological Products Technology Co., Ltd., Tianjin, China); 12-myristate-13acetate (PMA) (Sigma-Aldrich, Darmstadt, Germany); dichloro-dihydro-fluorescein diacetate (DCFH-DA) (Shanghai Biyuntian Biotechnology Co., Ltd., Shanghai, China); Hoechst (Shanghai Biyuntian Biotechnology Co., Ltd.); and SYTOX Green (Thermo Fisher Technology Co., Ltd., Waltham, MA, USA).

\section{Instruments}

The following instruments were used in this study: SpectraMax M5 Multifunctional Microplate Reader (Molecular Devices, LLC, San Jose, CA, USA); desktop high-speed refrigerated centrifuge (Beckman Coulter, Brea, CA, USA); inverted fluorescence microscope (Olympus, Shinjuku, Tokyo, Japan); and 96-well plate (Wuxi Nice Biotech Limited, Wuxi, Jiangsu, China).

\section{Study methods}

\section{Separation of neutrophils from human peripheral blood samples}

We collected $10 \mathrm{~mL}$ of venous peripheral blood from each healthy blood donor and added it to a centrifuge 
tube containing neutrophil separation solution, then it was centrifuged at $500 \mathrm{~g}$ for $35 \mathrm{~min}$ at room temperature. After centrifugation, 2 circular milky white cell layers appeared in the centrifuge tube: the upper cell layer was mononuclear cells and the lower cell layer was neutrophils. The neutrophils were subsequently transferred to a $15 \mathrm{~mL}$ clean centrifuge tube with a pipette, and the excess neutrophil separation solution was washed away with $10 \mathrm{~mL} 1640$ culture medium, then the cells were treated with red blood cell lysate at $4{ }^{\circ} \mathrm{C}$ for $10 \mathrm{~min}$. After $10 \mathrm{~min}$, cells were centrifuged at $250 \mathrm{~g}$ for $10 \mathrm{~min}$ at room temperature, the supernatant was discarded, the cells were then resuspended in $5 \mathrm{~mL} 1640$ medium, then the above 2 steps were repeated. Lastly, cells were counted with an optical microscope for the next step of the experiment.

\section{Experiment groups}

The neutrophils were uniformly added to a 96-well plate and divided into a control group, PMA group, PMA + PG490 $(0.2 \mu \mathrm{M})$ group, and PG490 $(0.2 \mu \mathrm{M})$ group. All groups were incubated in a humidified atmosphere of $5 \% \mathrm{CO}_{2}$ at $37^{\circ} \mathrm{C}$. The PG490 group and PMA + PG490 group were pre-treated with PG490 at their corresponding concentration for $30 \mathrm{~min}$. Next, all groups were treated with PMA (25 nM).

\section{NETs detection}

Cells were cultured according to method 2.2 and incubated with PMA (25 $\mathrm{nM}$ ) for $3 \mathrm{~h}$. Then SYTOX Green working fluid $(1 \mu \mathrm{M})$ was added into cells according to the instructions of the kit in the dark, and incubated for $15 \mathrm{~min}$. After that, phosphate-buffered saline (PBS; $1 \times$ ) was added to each well and mixed gently, and the samples were placed on ice. Cells were observed by fluorescence microscopy, collected the cells, and analyzed with flow cytometry.

\section{NETs immunofluorescence staining}

The cells were cultured according to method 2.2 and incubated with PMA (25 nM) for $3 \mathrm{~h}$. At the end of the incubation, cells were incubated with $100 \mu \mathrm{L}$ SYTOX Green stain $(100 \mathrm{nM})$ for $30 \mathrm{~min}$ at $37^{\circ} \mathrm{C}$, then the staining solution was discarded. Cells were incubated with $100 \mu \mathrm{L}$ Hoechst staining solution for $15 \mathrm{~min}$ at room temperature, Washed with PBS, then observed and analyzed by fluorescence microscopy.

\section{ROS detection}

At the end of the incubation for $30 \mathrm{~min}$ according to method 2.2, DCFH-DA was diluted with serum-free 1640 medium at the ratio of 1:1,000 with final concentration of $10 \mu \mathrm{M}$. The original cell culture medium was removed, then cells were incubated with $10 \mu \mathrm{L}$ DCFH-DA in an incubator at $37^{\circ} \mathrm{C}$ for $30 \mathrm{~min}$ in the dark, mixed upside down every 3 min to ensure the probe had full contact with the cells, then washed 3 times with PBS. Finally, ROS level was detected by flow cytometry and multifunction microplate reader.

\section{Statistical analysis}

Data were expressed as the mean \pm standard deviation (SD). The software SPSS 21.0 (IBM Corp., Armonk, NY, USA) was used for statistical analysis and PrismGraph 5.0 software (GraphPad Software, San Diego, CA, USA) was used for graphing. Data were analyzed using the ShapiroWilk Test for Gaussian distribution of the data, and by the Kruskal-Wallis test and Dunn's multiple comparison test for difference of neutrophil count and percentage between groups. Values of $\mathrm{P}<0.05$ were considered statistically significant.

\section{Results}

\section{Triptolide could inbibit the formation of NETS}

After the neutrophils from healthy blood donors had been treated with triptolide, PMA intervention for $3 \mathrm{~h}$ induced cell death. Compared with the PMA group, the average fluorescence intensity of SYTOX Green in the PG490 + PMA group, as detected by a multifunctional microplate reader, was significantly decreased (Figure 1), suggesting that PG490 can reduce the level of NETs. Similar results were observed by fluorescence microscope, whereby the number of NETs-generating cells represented by Hoechst and SYTOX Green co-labeling in the PMA group was significantly more than that in PG490 + PMA group (Figure 2). This indicated that triptolide can inhibit the formation of NETs.

\section{PG490 could not inhibit PMA-induced ROS level in neutrophils}

Neutrophils were pretreated with triptolide, and were then treated with PMA for $1 \mathrm{~h}$ to induce cell death. Intracellular ROS were labeled by fluorescence, with fluorescence intensity then measured by multifunctional microplate reader and flow cytometry. The results showed that compared with the control group, the fluorescence intensity 
of the PMA group was significantly increased, while there was no significant difference between PMA group and PG490 + PMA group (Figures 3,4). This confirmed that triptolide could downregulate neutrophil death, but it did not regulate PMA-induced neutrophil ROS level.

\section{Discussion}

In TCM, Tripterygium wilfordii Hook F has been widely used in a variety of rheumatoid immune diseases such as RA, Behçet's disease, SLE, nephrotic syndrome, and other diseases closely related to cell death. With the substantial development of modern medicine, people have found that Tripterygium wilfordii Hook $\mathrm{F}$ also has a therapeutic effect on leukemia. In 1972, one of the active components,

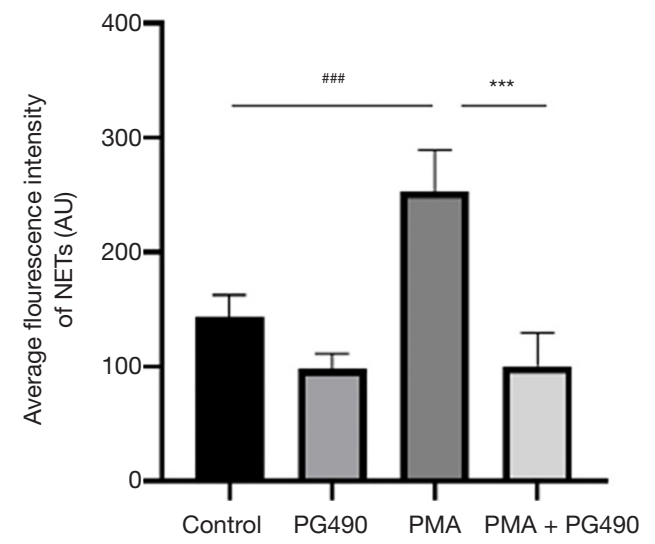

Figure 1 PG490 inhibits neutrophil NETs formation. ${ }^{\# \#} \mathrm{P}<0.001$ for comparisons between control group and PMA group; compared PMA group and PG490 groups, ${ }^{* *} \mathrm{P}<0.001$. NETs, neutrophil extracellular traps; PMA, 12 -myristate-13-acetate; PG490, triptolide. triptolide, was isolated from Tripterygium wilfordii Hook $\mathrm{F}$ in order to find drugs for treatment of leukemia (18). Furthermore, copious research has been conducted on its pharmacological effects, including side effects, to make enable its successful use as a clinical drug (19). In 2019, the team of Professor Luyong Zhang and Professor Zhenzhou Jiang discovered that programmed necrosis played an important role in liver toxicity of triptolide (20), while programmed necrosis was a critical factor for the formation of NETs (13).

Neutrophil death by NETs involves the release of a variety of inflammatory substances such as DNA, histones, elastase, and so on $(21,22)$. Current studies have found that these inflammatory substances are important source of autoantigens (23), and they are important factors of rheumatic immune diseases such as SLE, RA, and vasculitis

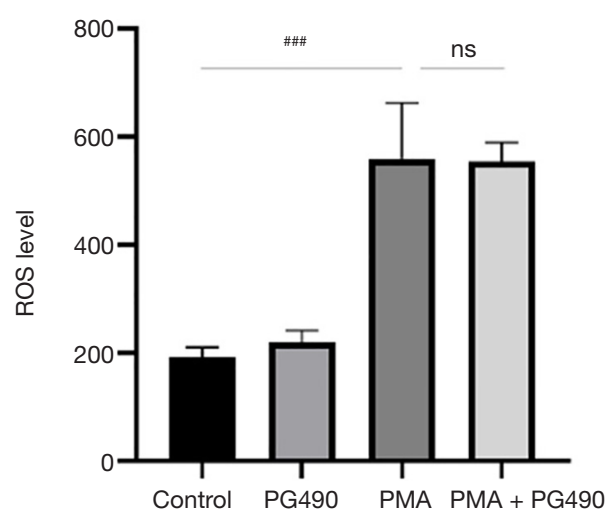

Figure 3 The effect of PG490 on the ROS production analyzed by SpectraMax M3 fluorescent plate reader. ${ }^{\# \# \#} \mathrm{P}<0.001$ for comparisons between control group and PMA group; compared PMA group and PG490 groups. ns, not significant; ROS, reactive oxygen species; PG490, triptolide; PMA, 12-myristate-13-acetate.
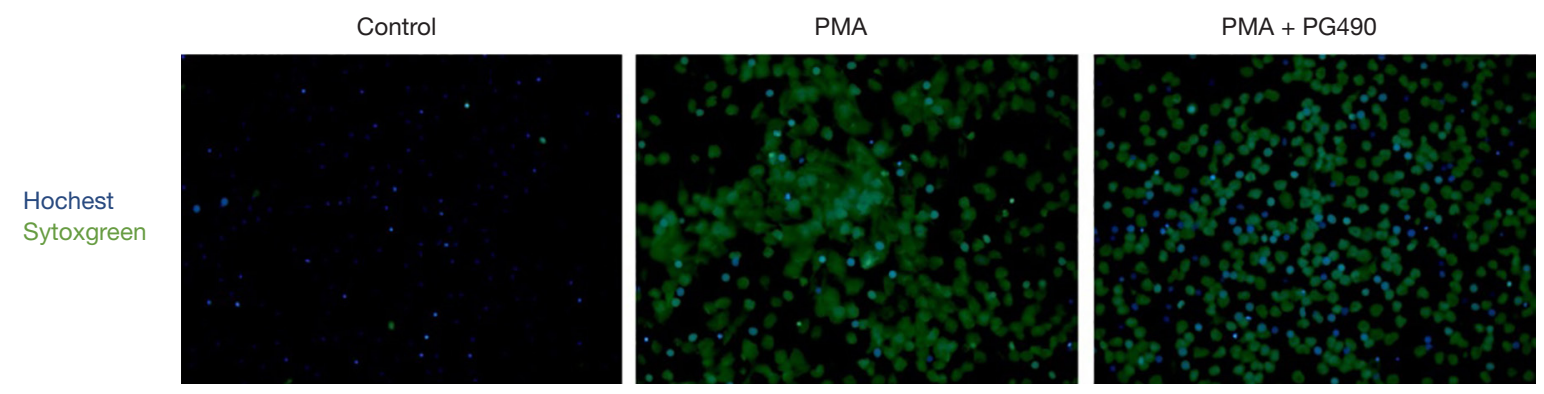

Figure 2 The effect of PG490 on the formation of NETs, analyzed by fluorescence microscope ( $\times 100)$. PMA, 12 -myristate-13-acetate; PG490, triptolide; NETs, neutrophil extracellular traps. 
A

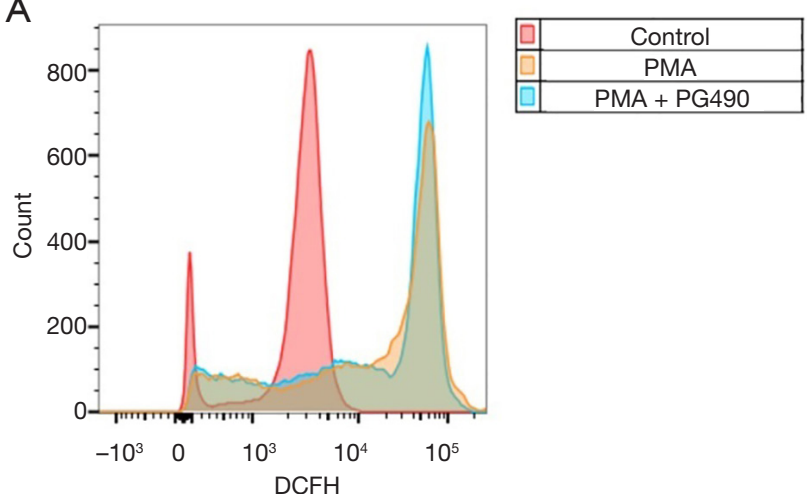

B

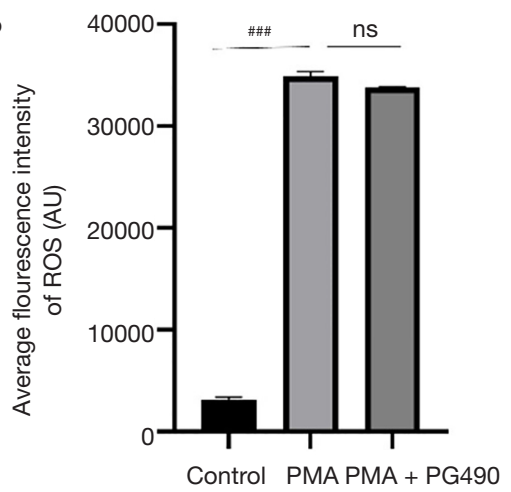

Figure 4 The effect of PG490 on the ROS production analyzed by flow cytometry. ${ }^{\# \#+} \mathrm{P}<0.001$ for comparisons between control group and PMA group; compared the PMA group and PG490 groups. ns, not significant; ROS, reactive oxygen species; PMA, 12-myristate-13-acetate; PG490, triptolide.

$(11,24,25)$. Therefore, inhibition of NETs production is one of the important targets for the treatment of rheumatic immune diseases.

In our study, we investigated whether triptolide could inhibit the production of NETs induced by PMA in neutrophils in vitro, which revealed that triptolide had a significant inhibitory effect, and it could protect cells from death in certain extent.

We also measured ROS level, an important factor involved in the formation of NETs, and the results showed that triptolide did not significantly reduce the ROS in the formation of NETs in human neutrophils, which was different from the results of triptolide acting on macrophages (4). Similarly, in previous studies on mouse peritoneal neutrophils (17), it has found that triptolide could inhibit the production of NETs and ROS. This indicated that triptolide may have different mechanisms for different human cells and between human and mouse neutrophils. This finding may provide some clues for future research on the mechanism of NETs.

The NETs release a variety of inflammatory substances, up-regulate the immune response, and are closely related to the occurrence and development of rheumatic immune diseases and tumors $(15,26-28)$. Due to various factors, we had not been able to analyze the specific effects of triptolide on these diseases by inhibiting the formation of NETs, including many indications of Tripterygium wilfordii Hook F. As one of the pathogenic factors of these diseases, the inhibition of the formation of NETs will definitely play a positive role in the treatment of the associated diseases.

In general, our study has provided a new idea for the study of the mechanism of triptolide on different immune cells and the specific mechanism for the treatment of rheumatoid diseases and tumor related diseases, and also provided a research basis for the clinical use of triptolide.

\section{Conclusions}

We demonstrated that PG490 inhibits the production of NETs in vitro, which is not associated with the level of cellular ROS.

\section{Acknowledgments}

Funding: This study was supported by the National Natural Science Foundation of China (81774038), the Guangdong Province Natural Science Foundation (2021A1515011672), and the President's Foundation of Integrated Traditional Chinese and Western Medicine Hospital of Southern Medical University (1201902002).

\section{Footnote}

Reporting Checklist: The authors have completed the MDAR reporting checklist. Available at https://dx.doi. org/10.21037/atm-21-3522

Data Sharing Statement: Available at https://dx.doi. org/10.21037/atm-21-3522

Conflicts of Interest: All authors have completed the ICMJE uniform disclosure form (available at https://dx.doi. org/10.21037/atm-21-3522). Haiyu Guan, Lifen Xie, Zhenzhen Ji, Jieying Qi, and Xiaoli Nie report funding support from the National Natural Science Foundation 
of China (81774038), the Guangdong Province Natural Science Foundation (2021A1515011672), and the President's Foundation of Integrated Traditional Chinese and Western Medicine Hospital of Southern Medical University (1201902002). The other author has no conflicts of interest to declare.

Etbical Statement: The authors are accountable for all aspects of the work in ensuring that questions related to the accuracy or integrity of any part of the work are appropriately investigated and resolved. All procedures performed in this study involving human participants were in accordance with the Declaration of Helsinki (as revised in 2013). The study was approved by ethics committee of Integrated Hospital of Traditional Chinese Medicine and Western Medicine (No.: NFZXYEC-201906-Y1) and informed consent was taken from all the patients.

Open Access Statement: This is an Open Access article distributed in accordance with the Creative Commons Attribution-NonCommercial-NoDerivs 4.0 International License (CC BY-NC-ND 4.0), which permits the noncommercial replication and distribution of the article with the strict proviso that no changes or edits are made and the original work is properly cited (including links to both the formal publication through the relevant DOI and the license). See: https://creativecommons.org/licenses/by-nc-nd/4.0/.

\section{References}

1. Fan D, He X, Bian Y, et al. Triptolide Modulates TREM1 Signal Pathway to Inhibit the Inflammatory Response in Rheumatoid Arthritis. Int J Mol Sci 2016;17:498.

2. Jiang J, Song X, Yang J, et al. Triptolide Inhibits Proliferation and Migration of Human Neuroblastoma SH-SY5Y Cells by Upregulating MicroRNA-181a. Oncol Res 2018;26:1235-43.

3. Zhan H, Jin J, Liang S, et al. Tripterygium glycoside protects diabetic kidney disease mouse serum-induced podocyte injury by upregulating autophagy and downregulating beta-arrestin-1. Histol Histopathol 2019;34:943-52.

4. Bao X, Cui J, Wu Y, et al. The roles of endogenous reactive oxygen species and nitric oxide in triptolideinduced apoptotic cell death in macrophages. J Mol Med (Berl) 2007;85:85-98.

5. Yuan Z, Zhang $H$, Hasnat $M$, et al. A new perspective of triptolide-associated hepatotoxicity: Liver hypersensitivity upon LPS stimulation. Toxicology 2019;414:45-56.

6. Kolaczkowska E, Kubes P. Neutrophil recruitment and function in health and inflammation. Nat Rev Immunol 2013;13:159-75.

7. Jaillon S, Ponzetta A, Di Mitri D, et al. Neutrophil diversity and plasticity in tumour progression and therapy. Nat Rev Cancer 2020;20:485-503.

8. Wu C, Xu K, Wang Z, et al. Increased neutrophils and IL-17A in a rare organizing pneumonia secondary to extrapulmonary operation. Ann Transl Med 2019;7:344.

9. Sollberger G, Tilley DO, Zychlinsky A. Neutrophil Extracellular Traps: The Biology of Chromatin Externalization. Dev Cell 2018;44:542-53.

10. Brinkmann V, Reichard U, Goosmann C, et al. Neutrophil extracellular traps kill bacteria. Science 2004;303:1532-5.

11. Lee KH, Kronbichler A, Park DD, et al. Neutrophil extracellular traps (NETs) in autoimmune diseases: A comprehensive review. Autoimmun Rev 2017;16:1160-73.

12. Dwivedi N, Radic M. Citrullination of autoantigens implicates NETosis in the induction of autoimmunity. Ann Rheum Dis 2014;73:483-91.

13. Schreiber A, Rousselle A, Becker JU, et al. Necroptosis controls NET generation and mediates complement activation, endothelial damage, and autoimmune vasculitis. Proc Natl Acad Sci U S A 2017;114:E9618-25.

14. Skopelja-Gardner S, Jones JD, Rigby WFC. "NETtling" the host: Breaking of tolerance in chronic inflammation and chronic infection. J Autoimmun 2018;88:1-10.

15. Jorch SK, Kubes P. An emerging role for neutrophil extracellular traps in noninfectious disease. Nat Med 2017;23:279-87.

16. Yu D, Liu Y, Zhou Y, et al. Triptolide suppresses IDH1mutated malignancy via Nrf2-driven glutathione metabolism. Proc Natl Acad Sci U S A 2020;117:9964-72.

17. Huang G, Yuan K, Zhu Q, et al. Triptolide inhibits the inflammatory activities of neutrophils to ameliorate chronic arthritis. Mol Immunol 2018;101:210-20.

18. Kupchan SM, Court WA, Dailey RG, Jr., et al. Triptolide and tripdiolide, novel antileukemic diterpenoid triepoxides from Tripterygium wilfordii. J Am Chem Soc 1972;94:7194-5.

19. Xi C, Peng S, Wu Z, et al. Toxicity of triptolide and the molecular mechanisms involved. Biomed Pharmacother 2017;90:531-41.

20. Huo J, Yu Q, Zhang Y, et al. Triptolide-induced hepatotoxicity via apoptosis and autophagy in zebrafish. J Appl Toxicol 2019;39:1532-40.

21. Papayannopoulos V. Neutrophil extracellular traps in 
immunity and disease. Nat Rev Immunol 2018;18:134-47.

22. Zawrotniak M, Rapala-Kozik M. Neutrophil extracellular traps (NETs) - formation and implications. Acta Biochim Pol 2013;60:277-84.

23. Castanheira FVS, Kubes P. Neutrophils and NETs in modulating acute and chronic inflammation. Blood 2019;133:2178-85.

24. Frangou E, Chrysanthopoulou A, Mitsios A, et al. REDD1/autophagy pathway promotes thromboinflammation and fibrosis in human systemic lupus erythematosus (SLE) through NETs decorated with tissue factor (TF) and interleukin-17A (IL-17A). Ann Rheum Dis 2019;78:238-48.

25. Khandpur R, Carmona-Rivera C, Vivekanandan-Giri A, et al. NETs are a source of citrullinated autoantigens and stimulate inflammatory responses in rheumatoid arthritis.

Cite this article as: Guan H, Xie L, Ji Z, Song R, Qi J, Nie X. Triptolide inhibits neutrophil extracellular trap formation. Ann Transl Med 2021;9(17):1384. doi: 10.21037/atm-21-3522
Sci Transl Med 2013;5:178ra40.

26. Nakazawa D, Masuda S, Tomaru U, et al. Pathogenesis and therapeutic interventions for ANCA-associated vasculitis. Nat Rev Rheumatol 2019;15:91-101.

27. Frangou E, Vassilopoulos D, Boletis J, et al. An emerging role of neutrophils and NETosis in chronic inflammation and fibrosis in systemic lupus erythematosus (SLE) and ANCA-associated vasculitides (AAV): Implications for the pathogenesis and treatment. Autoimmun Rev 2019;18:751-60.

28. Park J, Wysocki RW, Amoozgar Z, et al. Cancer cells induce metastasis-supporting neutrophil extracellular DNA traps. Sci Transl Med 2016;8:361ra138.

(English Language Editor: J. Jones) 